\title{
Adaptive Modulation Spectral Filtering for Improved Electrocardiogram Quality Enhancement
}

\author{
Diana P Tobón and Tiago H Falk
}

INRS-EMT, University of Québec, Montréal, QC, Canada

\begin{abstract}
Advances in portable electrocardiogram (ECG) monitoring devices has allowed for new cardiovascular applications to emerge beyond diagnostics, such as stress detection, sleep disorder characterization, mood recognition, activity surveillance, or fitness monitoring, to name a few. Such devices, however, are prone to artifacts, particularly due to movement, thus rendering heart rate and heart rate variability (HRV) metrics useless. To address this issue, this paper proposes a new ECG quality enhancement algorithm based on filtering in the so-called spectro-temporal (or modulation spectral) domain. Our experiments show that this new signal representation accurately separates ECG signal and noise components, thus allowing for adaptive filtering to improve signal quality even in extremely noisy settings. Experimental results show the proposed algorithm outperforming a state-of-the-art wavelet-based enhancement algorithm in terms of signal-to-noise ratio improvement, as well as ECG kurtosis; the latter has been widely used in the literature as an ECG quality index. The obtained findings suggest that the proposed algorithm can be used to enhance the quality of wearable ECG monitors even in extreme conditions, thus can play a key role in athletic peak performance training/monitoring.
\end{abstract}

\section{Introduction}

The increased risk of cardiovascular diseases, stress, hypertension, obesity, sleep disorders, and depression has motivated the use of portable (wearable) electrocardiogram (ECG) monitors for health diagnosis, but other market segments beyond medical applications are also emerging [1]. Low-cost, portable ECG devices, however, have been shown to be sensitive to numerous artifacts, including muscle contractions, baseline wander, and movement [2], thus lowering signal quality and, ultimately, hampering heart rate and heart rate variability (HRV) analyses [3]. To overcome this limitation, ECG quality enhancement algorithms are drastically needed that can operate under a wide range of noise levels.
Typically, three main methods have been explored in the past for ECG quality enhancement: filtering, empirical mode decomposition (EMD), or wavelet shrinkage. In [4], for example, ECG denoising was done using multiple iterations of a moving average filter and gains could be seen for noisy signals with a in signal-to-noise ratio (SNR) of around $10 \mathrm{~dB}$. In [5], in turn, nonlinear Bayesian filtering was performed and tested on signals corrupted by white and coloured gaussian noises; some filter stability issues were reported in extremely noisy settings and in the presence of arrhythmias. EMD-based algorithms, such as the one proposed in [6], rely on removal of mode functions mostly affected by the artifacts. Lastly, waveletbased algorithms are the most popular and works such as [7] and [8] rely on principles of shrinkage/thresholding. For wavelet-based algorithms, several parameters, such as mother wavelet, number of decomposition levels, thresholding selection rule, and thresholding method need to be optimized beforehand in an ad-hoc manner.

Here, we overcome these limitations by proposing a new algorithm based on adaptive filtering in the spectrotemporal domain, also known as modulation spectral domain. This signal representation characterizes the rate-ofchange of ECG spectral components, which was recently shown to differ from the rate-of-change of artifactual components, and to be independent of cardiac signal abnormalities [9]. By utilizing two invertible transforms to obtain the signal representation, filtering can be performed in the modulation spectral domain and the enhanced signal can be reconstructed. Such an approach has been used successfully for speech enhancement [10]. Experiments with synthetic ECG corrupted by realistic artifacts show the proposed algorithm outperforming a wavelet-based benchmark in terms of SNR and ECG kurtosis improvement; the latter being a widely-used quality index $[11,12]$.

\section{Methods and materials}

In this section, we explain the signal processing steps to calculate the ECG modulation spectrum, the modulation spectral filtering scheme, as well as the ECG database, the benchmark algorithm, and the performance metrics used. 


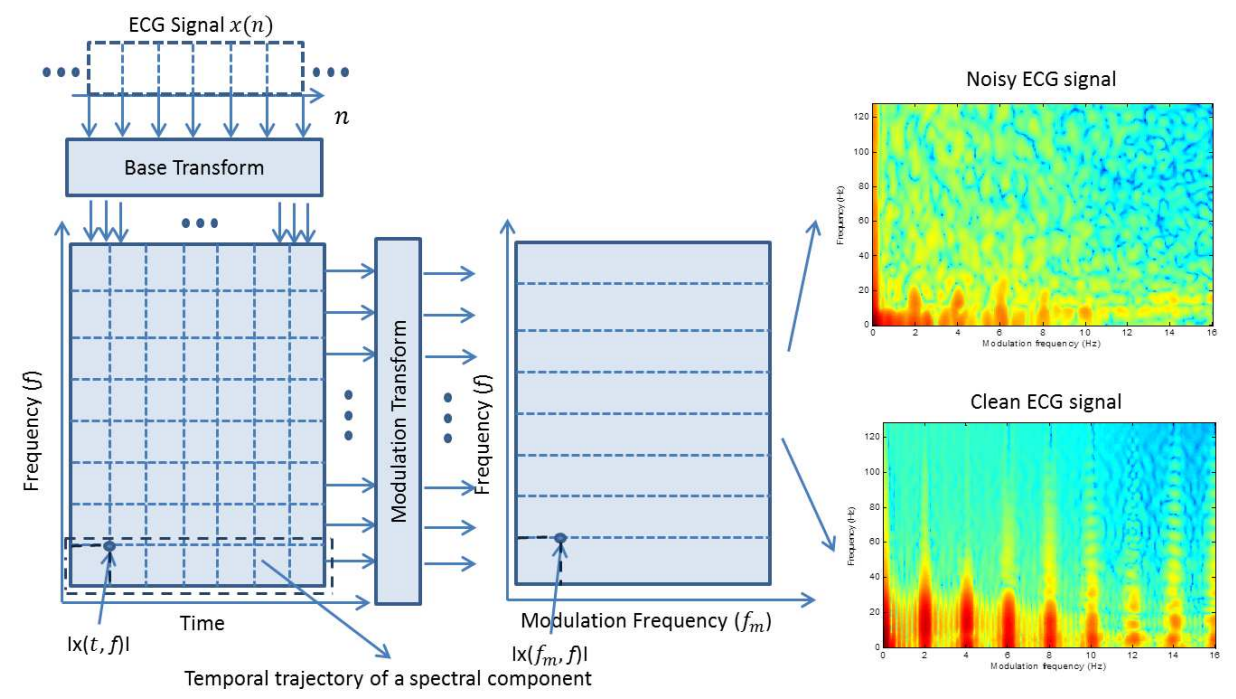

Figure 1. Signal processing steps to calculate the ECG modulation spectrum. Modulation spectrograms corresponds to a noisy ECG signal with $120 \mathrm{bpm}$ and an $S N R=-10 \mathrm{~dB}$ at the top, and its clean counterpart at the bottom.

\subsection{Modulation spectrum and filtering}

The processing steps required to obtain the ECG modulation spectral signal representation are depicted by Figure 1. First, the ECG signal (here sampled at $256 \mathrm{~Hz}$ ) is segmented using a 32 -point sine window with $75 \%$ overlap and transformed to the conventional time-frequency domain $(x(t, f))$ using a 512-point Fast-Fourier transform (FFT). Spectral magnitude components are then also segmented over time using a 128-point window with $75 \%$ overlap. A 512-point FFT across the time axis is performed to result into a final frequency-frequency representation $\left(x\left(f_{m}, f\right)\right)$ known as "modulation spectrum," where $f$ denotes conventional frequency and $f_{m}$ modulation frequency, both represented in $\mathrm{Hz}$.

As can be seen from the right-hand side of Fig. 1 (bottom), for a clean ECG signal, the representation is comprised (along the $f_{m}$ axis) of a central frequency, corresponding to the heart rate (in the case of the figure, 120 beats per minute, $\mathrm{bpm}$ ), its harmonics, as well as a component at $f_{m}=0 \mathrm{~Hz}$ corresponding to stationary components of the ECG signal. Along the conventional frequency axis, most of the energy is concentrated below $f<40 \mathrm{~Hz}$, as expected. The top right-hand plot of the figure, on the other hand, shows the effects of noise on the signal representation. At an SNR of -10dB, increased frequency components beyond $f=40 \mathrm{~Hz}$ can be seen at $f_{m}=0 \mathrm{~Hz}$, thus suggesting an increase in stationary noise, as well as increased modulation spectral energy between the main lobe and its harmonics (along the $f_{m}$ axis).

Given these insights, we propose the use of a series of adaptive bandpass filters in the modulation spectral domain. Bandpass filters are centred at the main lobe and sev- eral of its harmonics. The choice of windows and overlap used herein, inspired by the work in [9], result in a modulation spectral representation with maximum $f_{m}=16$ $\mathrm{Hz}$, thus the number of bandpass filters used depends on the signal's measured heart rate and range from 5-10 harmonics in the experiments described herein. To find the signal's instantaneous heart rate, we search for maximum modulation energy between $0 \leq f \leq 40 \mathrm{~Hz}$ and $0.8 \leq f_{m} \leq 3.3 \mathrm{~Hz}$, thus corresponding to heart rates between $50 \leq H R \leq 200 \mathrm{bpm}$. Since ECG components at $f_{m}=0 \mathrm{~Hz}$ were severely affected by noise, they are discarded completely at the cost of imperfect reconstruction of the ECG signal. While this can lead to issues with diagnostics where perfect QRS complexes are needed, for the task at hand (i.e., measure HR/HRV metrics) it was not a factor. Alternate strategies on how to handle this lowpass stationary components are still needed for diagnostics.

The signal processing steps for the proposed spectrotemporal filtering are depicted in Figure 2. The shortterm spectral component is denoted by $s(f, m)$, where $f=1, \ldots, N$ ( $f_{t h}$ frequency bin with $\mathrm{N}$ total number of frequency bands), and $m=1, \ldots, T$ ( $m_{t h}$ time step with $\mathrm{T}$ total number of time steps). Then, a Fast-Fourier transform is taken where the $|s(f, m)|$ magnitude for each frequency bin is filtered up to $f=40 \mathrm{~Hz}$ and $<s(f, m)$ phase is compensated. Each modulation processing bin has a bank of B adaptive linear-phase Finite Impulse Response (FIR) bandpass filters with length 432 to generate $\left|\hat{s}_{b}(f, m)\right|$. The bandwidth for the modulation bandpass filters was taken as $0.625 \mathrm{~Hz}$ based on insights from [3]. As mentioned above, the number of bandpass filters (B) is adapative to the signal's measured heart rate. After filtering, the 


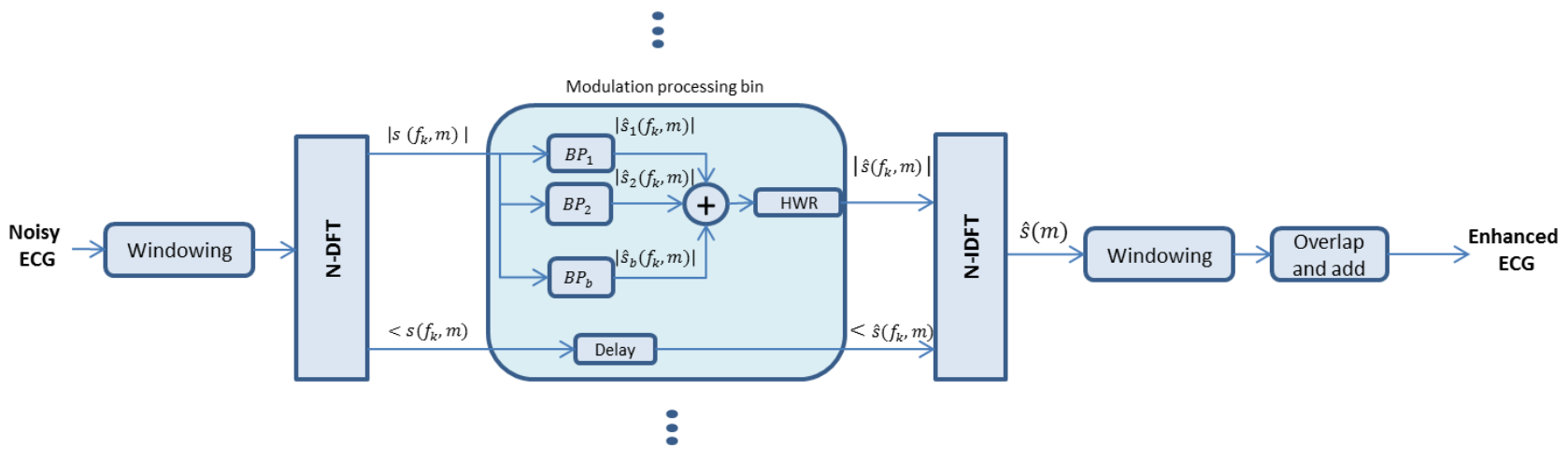

Figure 2. Signal processing steps used in the proposed modulation filtering method.
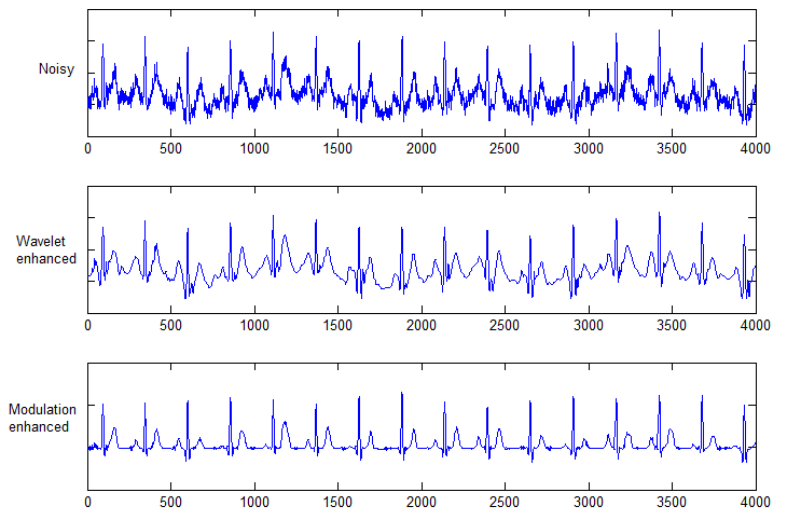

Figure 3. Noisy and enhanced ECG signals (60 bpm).

$\left|\hat{s}_{b}(f, m)\right|$ are added and compensated using a half wave rectification (HWR) due to negative power spectral values that can result from low frequency removal. Moreover, the phase is delayed by 216 samples resulting in $\angle \hat{s}(f, m)$. Finally, the processed magnitudes and phases from the $\mathrm{N}$ bins are transformed via an inverse Fourier transform into $\hat{s}(m)$ to be then windowed and overlap-added to obtain an enhanced ECG signal. For comparison purposes, Fig. 3 depicts an ECG signal with $60 \mathrm{bpm}$ and $S N R=0 d B$ before (top) and after wavelet (middle) and the proposed (bottom) modulation filtering schemes.

\subsection{ECG dataset}

Synthetic ECG signals with 2-minute duration were generated using the 'ecgsyn' Matlab function available within Physionet [13]. We created 700 signals ranging from 50 to $180 \mathrm{bpm}$ to cover tachycardia, bradycardia, and different activity levels such as sitting, walking, and running. The signals were generated to include low frequency to high frequency ratio (LF/HF) between 0.5 and 8.9 randomly sampled to cover light-to-deep sleep, wake- fulness, myocardial infarction, and rapid eye movement [2]. The synthetic signals were contaminated with artifacts (i.e., electrode motion, muscle artifacts, and baseline wander noise) taken from MIT-BIH Noise Stress Test Database [13] at five different signal-to-noise (SNR) levels such as $-10,-8,-5,0$, and $5 \mathrm{~dB}$. We also used pink and brownian noise to model observation noise and electrode movement artifacts respectively. Hence, we have 4900 signals for analysis, totalling 163 hours for testing.

\subsection{Benchmark algorithm and perfor- mance assessment metrics}

As mentioned above, wavelet-based denoising algorithms are the most popular, thus are used here to benchmark the proposed algorithm. A subset of 100 signals, each corrupted by the five SNR levels, was used to optimize benchmark algorithm parameters. It was found that the universal shrinkage method with soft thresholding, and a Daubechies mother wavelet with 8 decomposition levels resulted in the best performance on this subset. As such, this configuration was used in our experiments.

As figures of merit to gauge the advantages of the proposed algorithm, two metrics were used: (1) the kurtosis $(\kappa)$ measured within a window around the $\mathrm{R}$ peaks in the QRS complexes, and (2) the SNR improvement post enhancement. According to $[11,12]$ higher kurtosis values correspond to improved quality and values of $\kappa \geq 5$ typically correspond to good quality ECGs.

\section{Results and discussion}

Table 1 shows $\kappa$ and post-enhanced SNRs for the proposed and benchmark algorithms before and after enhancement. It can be seen that the average SNR improvement for noisier signals $(\mathrm{SNR}=-10,-8,-5$, and $0 \mathrm{~dB})$ with the proposed algorithm was $9 \mathrm{~dB}$ compared to $4 \mathrm{~dB}$ with the benchmark. SNR improvements increased for noisier sig- 
Table 1. Performance comparison between proposed and benchmark algorithms before and after enhancement.

\begin{tabular}{cccccc}
\hline \hline \multicolumn{2}{c}{ Noisy } & \multicolumn{2}{c}{ Proposed } & \multicolumn{2}{c}{ Benchmark } \\
\hline Input SNR (dB) & $\kappa$ & Post-SNR (dB) & $\kappa$ & Post-SNR (dB) & $\kappa$ \\
-10 & $3.1 \pm 0.0$ & $1.4 \pm 1.2$ & $6.2 \pm 1.5$ & $-2.3 \pm 0.1$ & $3.0 \pm 0.1$ \\
-8 & $3.4 \pm 0.0$ & $2.5 \pm 1.3$ & $7.3 \pm 1.6$ & $-2.0 \pm 0.2$ & $3.5 \pm 0.1$ \\
-5 & $4.2 \pm 0.2$ & $3.9 \pm 1.2$ & $8.6 \pm 1.7$ & $-1.2 \pm 0.2$ & $4.4 \pm 0.3$ \\
0 & $5.6 \pm 0.4$ & $5.5 \pm 0.9$ & $10.0 \pm 1.7$ & $1.2 \pm 0.2$ & $5.8 \pm 0.6$ \\
5 & $6.3 \pm 0.6$ & $6.4 \pm 0.7$ & $10.7 \pm 1.7$ & $5.0 \pm 0.2$ & $6.5 \pm 0.8$ \\
\hline \hline
\end{tabular}

nals. Moreover, $\kappa$ measurements were higher with the proposed algorithm across all tested SNR levels, thus suggesting improved quality over the benchmark. An average $\kappa$ increase over the noisy signal of 4 was obtained with the proposed algorithm, thus outperforming the subtle increase of 0.1 obtained with the benchmark. Using the $\kappa$ threshold of 5 suggested in $[11,12]$ to indicate good quality ECG, this could be achieved with the proposed solution even in extremely noisy cases $(\mathrm{SNR}=-10 \mathrm{~dB})$, thus comparing favourably against the benchmark which only achieved this at an SNR $=0 \mathrm{~dB}$. Overall, the proposed solution had a higher $\kappa$ standard deviation, which seemed to be around 1.6 irrespective of the tested SNR. Despite the higher deviation, the $\kappa$ increases with the proposed algorithm were significantly higher than those achieved with the benchmark across all SNR levels. Overall, the obtained findings suggest that the proposed algorithm may be better suitable for ECG quality enhancement, particularly in extremely noisy scenarios.

\section{Conclusion}

A new method to denoise ECG signals was proposed based on adaptive filtering in the modulation spectral domain. The algorithm was tested on 163 hours of ECG data of varying noise levels and shown to outperform a state-ofthe-art benchmark algorithm based on wavelet shrinkage in terms of SNR improvement and ECG kurtosis increase. The obtained findings suggest that the proposed technique is well suitable for lower-cost wearable ECG devices that can be highly contaminated by movement artifacts.

\section{References}

[1] Tobon DP, Falk TH, Maier M. Context awareness in WBANs: a survey on medical and non-medical applications. IEEE Wireless Communications 2013;20(4):30-37.

[2] Clifford GD, et al. ECG statistics, noise, artifacts, and missing data. Advanced Methods and Tools for ECG Data Analysis 2006;55-99.

[3] Tobon Vallejo D, Falk TH, Maier M. MS-QI: A Modulation Spectrum-Based ECG Quality Index for Telehealth Applications. IEEE Transactions on Biomedical Engineering to appear;.
[4] Salih SK, Aljunid S, Aljunid SM, Maskon O. Adaptive filtering approach for denoising electrocardiogram signal using moving average filter. Journal of Medical Imaging and Health Informatics 2015;5(5):1065-1069.

[5] Sameni R, Shamsollahi MB, Jutten C, Clifford GD. A nonlinear Bayesian filtering framework for ECG denoising. Biomedical Engineering IEEE Transactions on 2007; 54(12):2172-2185.

[6] Kabir MA, Shahnaz C. Denoising of ECG signals based on noise reduction algorithms in EMD and wavelet domains. Biomedical Signal Processing and Control 2012;7(5):481489.

[7] Singh BN, Tiwari AK. Optimal selection of wavelet basis function applied to ECG signal denoising. Digital Signal Processing 2006;16(3):275-287.

[8] Zhang D. Wavelet approach for ECG baseline wander correction and noise reduction. In Engineering in Medicine and Biology Society, 2005. IEEE-EMBS 2005. 27th Annual International Conference of the. IEEE, 2005; 1212-1215.

[9] Tobon DP, Falk TH. Online ECG quality assessment for context-aware wireless body area networks. In IEEE 28th Canadian Conference on Electrical and Computer Engineering (CCECE), 2015. IEEE, 2015; 587-592.

[10] Falk TH, Stadler S, Kleijn WB, Chan WY. Noise suppression based on extending a speech-dominated modulation band. In Proc. INTERSPEECH. 2007; 970-973.

[11] Behar J, Oster J, Li Q, Clifford GD. ECG signal quality during arrhythmia and its application to false alarm reduction. Biomedical Engineering IEEE Transactions on 2013; 60(6):1660-1666.

[12] Li Q, Mark RG, Clifford GD. Robust heart rate estimation from multiple asynchronous noisy sources using signal quality indices and a kalman filter. Physiological measurement 2007;29(1):15.

[13] Goldberger AL, Amaral LA, Glass L, Hausdorff JM, Ivanov PC, Mark RG, Mietus JE, Moody GB, Peng CK, Stanley HE. Physiobank, physiotoolkit, and physionet components of a new research resource for complex physiologic signals. Circulation 2000;101(23):e215-e220.

Address for correspondence:

Tiago H. Falk

INRS-EMT, University of Quebec (suite 6900)

800 rue de la Gauchetiere O., Montreal, QC, H5A 1K6

falk@emt.inrs.ca 